\title{
Celecoxib attenuates liver steatosis and inflammation in non-alcoholic steatohepatitis induced by high-fat diet in rats
}

\author{
JING CHEN $^{1}$, DIANGANG LIU ${ }^{2}$, QIXUAN BAI ${ }^{3}$, JITAO SONG ${ }^{1}$, \\ JIA GUAN $^{1}$, JIE GAO ${ }^{1}$, BINGRONG LIU ${ }^{1}$, XIAO MA $^{1}$ and YAJU DU ${ }^{1}$ \\ ${ }^{1}$ Department of Gastroenterology, the Second Affiliated Hospital of Harbin Medical University, Harbin; \\ ${ }^{2}$ Department of General Surgery, XuanWu Hospital, Capital Medical University, Beijing; \\ ${ }^{3}$ Department of Gastroenterology, Civil Aviation General Hospital, Peking University, Beijing, P.R. China
}

Received February 18, 2011; Accepted May 30, 2011

DOI: $10.3892 / \mathrm{mmr} .2011 .501$

\begin{abstract}
Cyclooxygenase-2 (COX-2) is involved in the process of non-alcoholic steatohepatitis (NASH). However, the role of the COX-2 inhibitor in NASH has not yet been elucidated. Therefore, in the present sudy, we investigated the role of celecoxib in a rat model of NASH induced by a high-fat diet (HFD). Wistar rats were administered HFD by gavage, and rats administered normal saline by gavage served as the controls. After 4 weeks of HFD feeding, the rats were treated with celecoxib $(20 \mathrm{mg} / \mathrm{kg} / \mathrm{day})$ or placebo for 4 weeks. At the end of 4 and 8 weeks, histological changes in the livers of the rats were analyzed using hematoxylin and eosin; blood was collected to detect biochemical indicators (serum aminotransferase and triglyceride). Liver triglyceride content was measured using the triglyceride E-test kit. The liver expression of $\mathrm{COX}-2$, nuclear factor- $\kappa$ enhancer binding protein $(\mathrm{NF}-\mathrm{kB}$ ) subunits p50 and p65 was measured by real-time reverse transcription-polymerase chain reaction and/or Western blotting. Infiltration of steatosis and inflammation in cells was observed in the livers after 4 weeks of HFD administration, and marked steatosis and inflammation was induced after 8 weeks. These histological changes were significantly attenuated after celecoxib treatment. Reduced serum alanine aminotransferase and triglyceride (TG) levels and TG content in the liver were observed in the HFD rats that received celecoxib. Moreover, celecoxib suppressed hepatic COX-2 messenger RNA and protein expression. The NF- $\kappa B$ subunit p50 and p65 protein levels in the HFD rats were also
\end{abstract}

Correspondence to: Dr Yaju Du, Department of Gastroenterology, the Second Affiliated Hospital of Harbin Medical University, 246 XueFu Road, Nangang, Harbin, Heilongjiang 150086, P.R. China E-mail: duyaju@medmail.com.cn

Abbreviations: NASH, non-alcoholic steatohepatitis; COX-2, cyclooxygenase-2; HFD, high-fat diet; NF- $\kappa \mathrm{B}$, nuclear factor- $\kappa$ enhancer binding protein

Key words: non-alcoholic steatohepatitis, non-alcoholic fatty liver disease, celecoxib, nuclear factor- $\kappa$ enhancer binding protein attenuated after celecoxib treatment. The results indicate that the induction of COX-2 occurs in association with NF- $\mathrm{KB}$ activation in HFD-induced NASH rats. Celecoxib may protect against the development of steatohepatitis induced by HFD.

\section{Introduction}

Cyclooxygenase (COX) catalyzes the rate limiting steps in prostaglandin (PG) synthesis. Mammalian cells express two distinct isoforms of COX, COX-1 and COX-2. COX-1 is constitutively expressed in many tissues and catalyzes the formation of cytoprotective PGs (1). COX-2 is rapidly induced in response to inflammation, and is predominantly responsible for the production of a variety of PGs, which may cause or protect against inflammation-induced pathology (2). COX-2 is expressed only at low levels in normal hepatocytes, whereas it is markedly increased in chronic hepatitis and liver cirrhosis $(3,4)$. It has been reported that the selective inhibitor of COX-2, celecoxib $(20 \mathrm{mg} / \mathrm{kg} / \mathrm{day})$, reduced hepatic fibrosis in both CCl4-, BDL- and TAA-treated rats (5-7).

Currently, inflammation is an aspect of non-alcoholic steatohepatitis (NASH) that has received considerable attention as a possible target for therapy. Drugs that inhibit COX-2 more than COX-1 cause fewer adverse effects, such as severe damage to the gastric tissues. The selective COX-2 inhibitor may therefore be a possible strategy for the treatment of non-alcoholic steatohepatitis (NASH). However, the literature regarding the effect of celecoxib on NASH animal models is limited. COX-2 is increased in mice with methionine- and choline-deficient diet (MCD)-induced steatohepatitis, and the COX-2 inhibitor could reverse the severity of hepatitis (8). However, the shortcomings of the MCD model should not be ignored. The major disadvantage is that the metabolic profile of MCD-fed mice is generally the converse of human NASH, which has decreased serum insulin and leptin levels, lower fasting blood glucose levels, is peripherally insulin sensitive, and has unchanged or increased serum adiponectin levels $(9,10)$. The high-fat diet (HFD)-induced model avoids this disadvantage and mimics human NASH features $(9,11)$. However, the effects of celecoxib on the HFD-induced NASH model are unkown.

HFD-induced steatohepatitis models were therefore used to investigate the potential role of COX-2 in the pathogen- 
esis of NASH. The direct effect of celecoxib on this animal model was evaluated. In the present study, we examined the changes of COX-2 and nuclear factor- $\kappa$ enhancer binding protein (NF- $\mathrm{kB}$ ) expression in HFD-induced NASH rats and conducted intervention studies to clarify the pathogenic significance of COX-2 up-regulation with the selective COX-2 inhibitor, celecoxib.

\section{Materials and methods}

Reagents. Celecoxib [4-(5-(4-methylphenyl)-3-(trifluoromethyl)1H-pyrazol-1-yl)benzene-sulfonamide] was a generous gift from the Pfizer Pharmaceutical Group (Groton, CT, USA). The antibodies against rat COX-2 (sc-7951), rat NF-кB p50 (sc-8414) and rat NF-kB p65 (sc-7151) were purchased from Santa Cruz Biotechnology (Santa Cruz, CA, USA).

Animal treatments. Male Wistar rats weighing 200-250 g were obtained from the Harbin Veterinary Research Institute (Harbin, China). All protocols and procedures were approved by the Animal Care and Use Committee of Harbin Medical University Health Science Center in accordance with National Institutes of Health guidelines. The animals were housed in an air-conditioned room at $23-25^{\circ} \mathrm{C}$ with a 12 -h dark/light cycle for 1 week before initiation of the experiment. All animals received humane care during the study with unlimited access to food and water.

Rats were randomly divided into a control group ( $\mathrm{n}=14)$ and NASH group $(\mathrm{n}=28)$. The rat model of NASH was induced by gavage with a HFD $(25 \%$ lard $+2 \%$ cholesterol $+0.5 \%$ sodium cholate $+25 \%$ Tween- 80 ) for 8 weeks. The control rats were administered normal saline by gavage. At the end of week 4 , some rats (control group $n=7$, NASH group $n=7$ ) were sacrificed under anesthesia and liver tissues were obtained and stored. The remaining HFD rats were then randomly treated with celecoxib $(20 \mathrm{mg} / \mathrm{kg} /$ day, $\mathrm{n}=10)$ or placebo $(0.5 \%$ carboxymethylcellulose, $n=10$ ) by gastric gavage for another 4 weeks. The dosage of celecoxib was based on the daily human dose, which was $400-800 \mathrm{mg} /$ day; previous studies in rats with this dosage has demonstrated the antifibrotic effect in rat livers (5).

At the end of the study, all rats were sacrificed after $12 \mathrm{~h}$ of fasting. Blood samples were collected for biochemical assays. The liver was removed and weighed after being rinsed with ice-cold saline, and sampled for histological study, RNA expression, and Western blot analysis.

Biochemical analyses. Blood biochemical parameters, including serum alanine aminotransferase (ALT) and triglyceride (TG) were assayed using an automatic biochemical analyzer (7600; HITEC, Japan). Liver TG was measured using the triglyceride E-test kit (Wako Pure Chemical Industries, Osaka, Japan).

Histological assessment. The liver samples were fixed in $10 \%$ formalin, then sliced into 4- to 6-mm pieces, dehydrated in ethanol, embedded in paraffin wax, sectioned $(5 \mu \mathrm{m}$ thick), and stained with hematoxylin and eosin. Steatosis and necroinflammatory grade were assessed as previously described (8). Liver histology was evaluated blindly by two independent liver pathologists and scored for hepatic steatosis (0, none; 1, 1-25\%; 2, 26-50\%; 3, 51-75\%; 4, 76-100\% hepatocytes affected) and necroinflammation ( 0 , none; 1 , mild; 2 , moderate; 3 , severe).

Quantification of hepatic messenger RNA ( $m R N A$ ) expression levels. Total RNA was extracted from whole liver using TRIzol reagent (Invitrogen, Carlsbad, CA, USA). Hepatic complementary DNA was used to quantify COX-2 mRNA levels via real-time polymerase chain reaction (RT-PCR) using SYBR-Green Master Mix (Applied Biosystems, Foster, CA, USA). To control for variations in the reactions, all PCRs were normalized to glyceraldehyde-3-phosphate dehydrogenase $(\mathrm{GAPDH})$. The specific primers used were as follows: GADPH, F: 5'CCTGCCAAGTATGATGACATCAAGA3'; R: 5'GTAGCCCAGGATGCCCTTTAGT3'. COX-2, F: 5'GATG CTATCTTTGGGGAGACC3'; R: 5'CCATAAGGCCTTTCAA GGAGA3'.

Western blot analysis. Liver tissue was homogenized in Tris- $\mathrm{HCl}$ (pH 7.4) buffer containing phenylmethylsulfonyl fluoride (PMSF), and total protein as determined using the BCA protein assay kit (Thermo, CA, USA). Protein $(30 \mu \mathrm{g})$ was subjected to $10 \%$ sodium dodecyl sulfate polyacrylamide gel electrophoresis, transferred to a polyvinylidene difluoride membrane (Millipore, Bedford, MA, USA) and probed with the primary antibodies against COX-2 $(1: 1,000)$, or $\beta$-actin (Sigma-Aldrich, St. Louis, MO, USA) $(1: 5,000)$. Nuclear protein $(50 \mu \mathrm{g})$ was electrophoresed through a $10 \%$ polyacrylamide gel, and immunoblots were incubated with primary antibodies against the NF-kB subunits p65 and p50 (1:500) (Santa Cruz Biotechnology).

Proteins were detected via Immobilon Western HRP Substrate (Millipore, Bedford, MA, USA), and bands were quantified via scanning densitometry using the SCAN Control (Scanco 1.lnk) imaging system. Individual levels of protein expression were normalized to $\beta$-actin.

Statistical analysis. The results are presented as the means \pm SD. Statistical tests were performed with SPSS software version 11.5 (SPSS Inc., Chicago, IL, USA). Differences between two groups were analyzed via the Student's t-test, and comparisons between more than 2 groups were analyzed by one-way analysis of variance to identify differences among the means. P-values of $<0.05$ were considered statistically significant.

\section{Results}

Celecoxib decreases serum $A L T$ and $T G$ levels in HFD-induced NASH rat models. At 8 weeks, HFD caused a significant increase in serum ALT and TG levels compared with normal rats (normal vs. HFD-placebo group, ALT: $64.61 \pm 13 \mathrm{IU} / \mathrm{ml}$ vs. $358.4 \pm 31 \mathrm{IU} / \mathrm{ml}$; TG: $0.78 \pm 0.38 \mathrm{mmol} / 1$ vs. $2.3 \pm 0.37 \mathrm{mmol} / 1$, respectively). The HFD plus celecoxib group showed significantly lower levels of serum ALT and TG than the HFD plus placebo group $(\mathrm{P}<0.01$ and $\mathrm{P}<0.05$, respectively) (Fig. 1A and B).

Celecoxib reverses hepatic steatosis and inflammation in HFD-induced NASH rat models. The control rats exhibited 

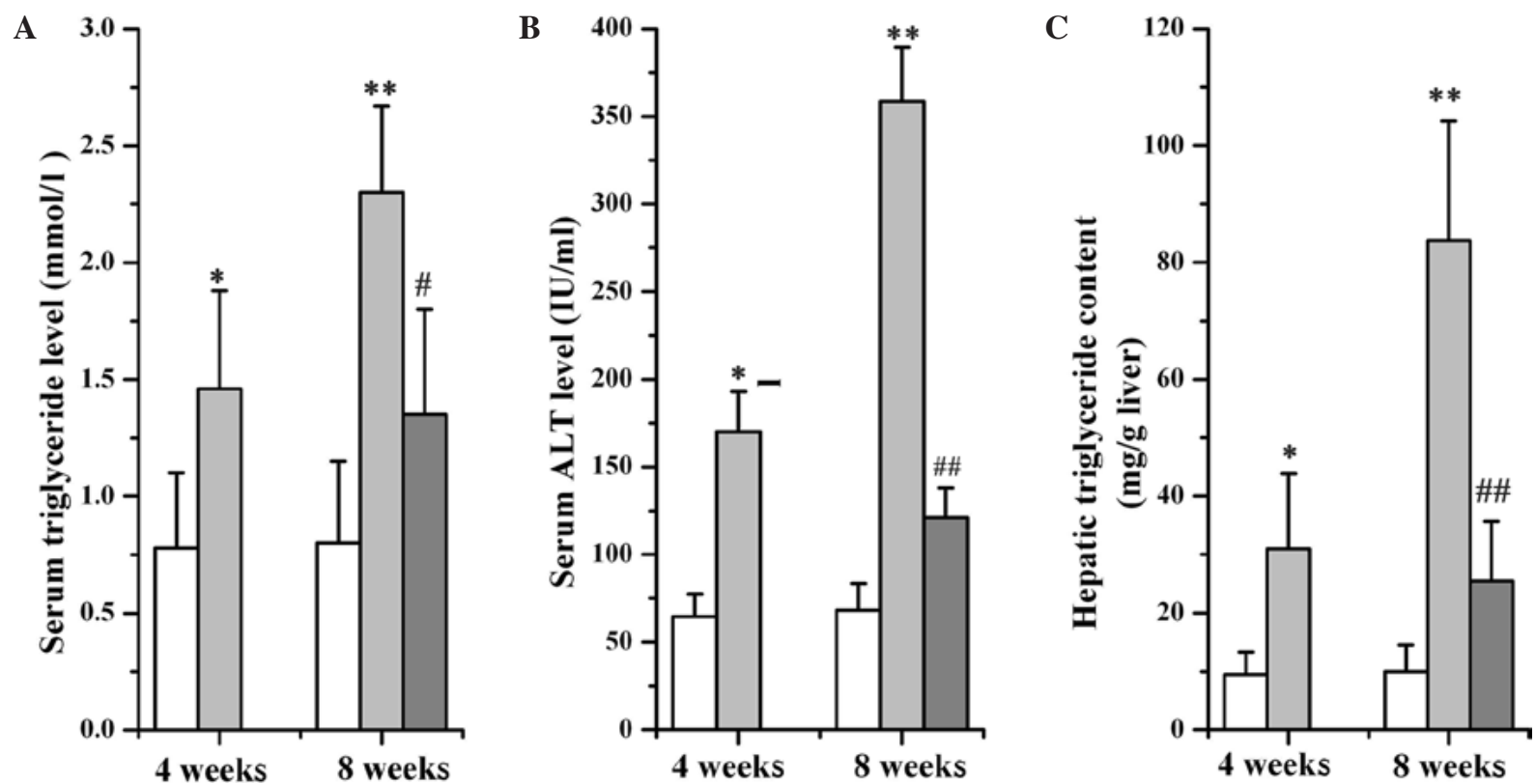

Figure 1. Effects of the HFD and treatment with celecoxib on serum ALT, triglyceride level and hepatic triglyceride expression in rats. Wistar rats were fed the control diet (white bars), HFD (gray bars), or the HFD with celecoxib $(20 \mathrm{mg} / \mathrm{kg} /$ day) (black bars) for 4 or 8 weeks. (A) Serum triglyceride level, (B) serum ALT levels, $(\mathrm{C})$ hepatic triglyceride content. Data are expressed as the means $\pm \mathrm{SD}$. ( $\mathrm{n}=7-10 /$ group). ${ }^{*} \mathrm{P}<0.05,{ }^{* *} \mathrm{P}<0.01$ (HFD vs. control). ${ }^{\#} \mathrm{P}<0.05$, ${ }^{\# \#} \mathrm{P}<0.01$ (HFD + celecoxib vs. HFD + placebo).
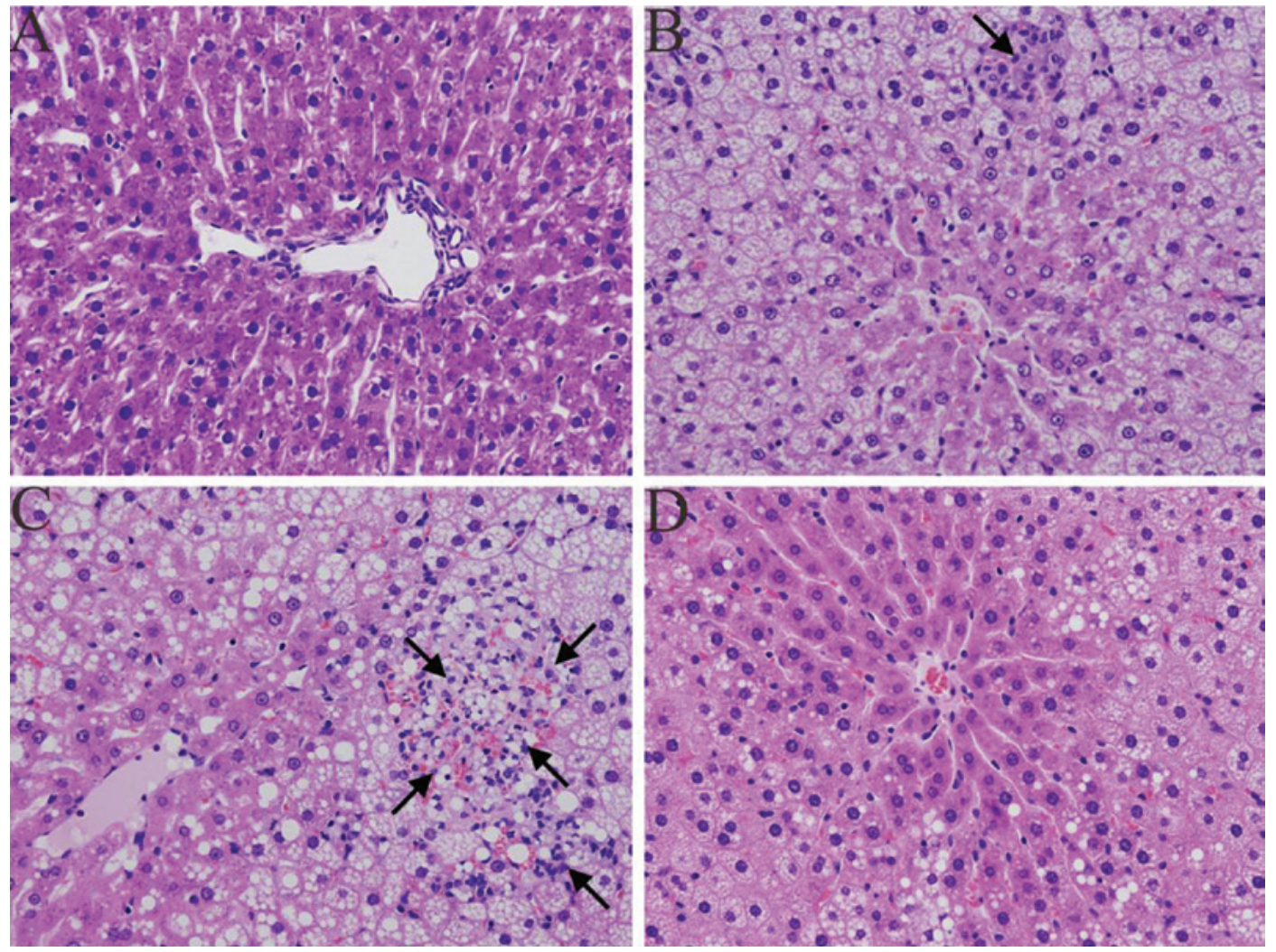

Figure 2. Histological change in the liver of HFD and celecoxib-treated rats (normal diet rats as controls). (A) Wistar rats fed the normal diet for 4 weeks showed normal liver histology. (B) When rats were fed the HFD for 4 weeks, fat droplets and small groups of inflammatory cells (arrow) were observed. (C) After 8 weeks of HFD feeding, the severity of steatohepatitis greatly increased. (D) Treatment of HFD rats with celecoxib for 4 weeks attenuated steatohepatitis (HE x40).

normal liver histology (Fig. 2A), when fed the HFD for 4 weeks; vesicular fat droplets and small groups of inflammatory cells (arrow) were observed (Fig. 2B). Significant hepatic steatosis and necroinflammation were induced by a HFD fed to rats for 8 weeks, without obvious fibrosis information (Fig. 2C). Celcecoxib (20 mg/kg/day) attenuated 
Table I. Effect of HFD and treatment with celecoxib on scores for hepatic steatosis and necroinflammatory lesions in rats.

\begin{tabular}{|c|c|c|c|c|c|}
\hline & \multicolumn{2}{|c|}{4 weeks } & \multicolumn{3}{|c|}{8 weeks } \\
\hline & Control & HFD & Control & HFD + Placebo & HFD + celecoxib \\
\hline Steatosis & $0.0 \pm 0.0$ & $2.5 \pm 0.6^{\mathrm{a}}$ & $0.0 \pm 0.0$ & $3.5 \pm 0.4^{\mathrm{a}}$ & $1.4 \pm 0.9^{\mathrm{b}}$ \\
\hline Necroinflammation & $0.0 \pm 0.0$ & $1.2 \pm 0.9^{\mathrm{a}}$ & $0.0 \pm 0.0$ & $2.8 \pm 0.4^{\mathrm{a}}$ & $1.0 \pm 0.5^{\mathrm{b}}$ \\
\hline
\end{tabular}

The values of hepatic steatosis and necroinflammation are expressed as the means $\pm \mathrm{SD}$ ( $\mathrm{n}=7-10 /$ group). ${ }^{\mathrm{P}}<0.05$ (HFD vs. control diet), ${ }^{\mathrm{b}} \mathrm{P}<0.05$ (HFD with placebo vs. HFD with celecoxib treatment).

A

\begin{tabular}{|c|c|c|c|c|}
\hline \multicolumn{2}{|c|}{4 weeks } & \multicolumn{3}{|c|}{8 weeks } \\
\hline Normal & NASH & Normal & NASH & Celecoxib-treated \\
\hline
\end{tabular}

B

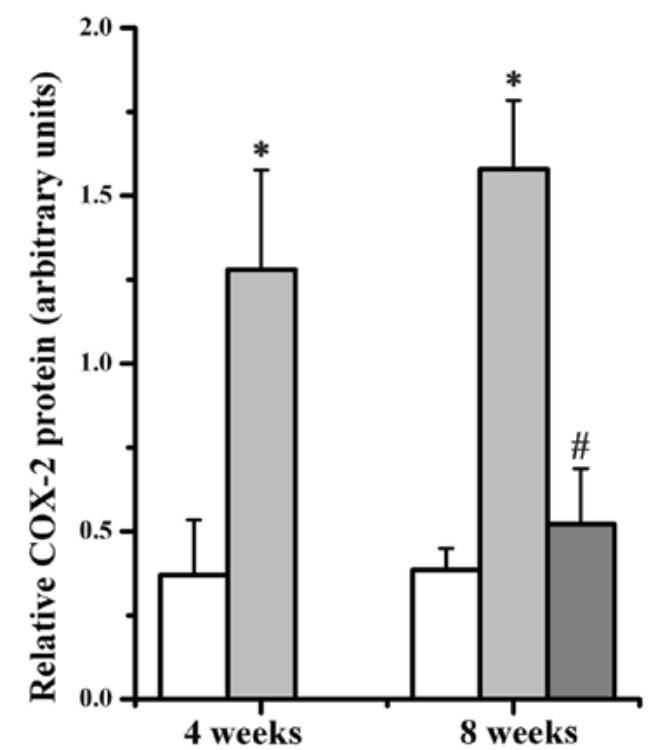

C

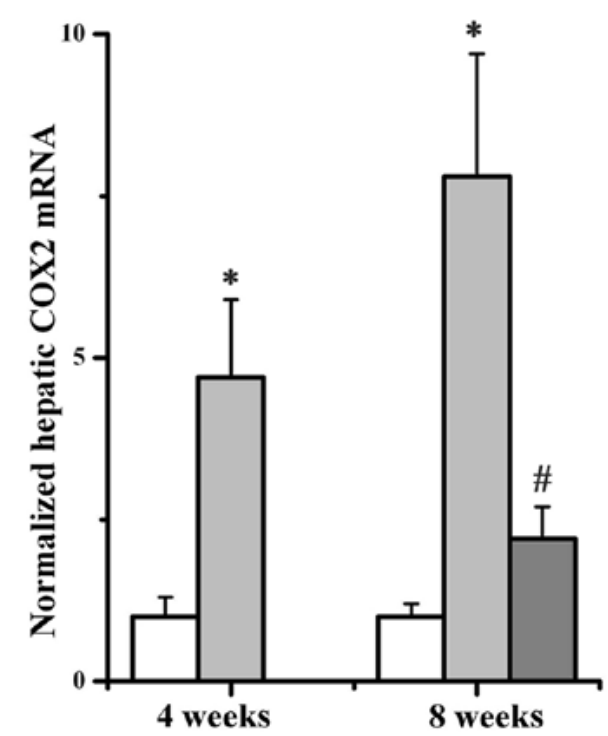

Figure 3. Effects of the HFD and treatment with celecoxib on hepatic COX-2 expression. Wistar rats were fed the control diet (white bars), HFD (gray bars), or HFD with celecoxib (20 mg/kg/day) (black bars) for 4 or 8 weeks. (A) Hepatic COX-2 and $\beta$-actin protein levels were determined via Western blotting, (B) bands were quantified, and COX-2 protein levels were expressed as relative amounts normalized to $\beta$-actin. (C) COX-2 mRNA values were normalized to the expression of GAPDH. Data are expressed as the means \pm SD. ${ }^{*} \mathrm{P}<0.05$ (HFD vs. control). ${ }^{\#} \mathrm{P}<0.05$ (HFD + celecoxib vs. HFD + placebo), ( $\mathrm{n}=7-10 /$ group).

hepatic steatiosis and necroinflammation in rats (Fig. 2D). Liver histology score demonstrated that celecoxib treatment significantly decreased hepatic steatosis (HFD + placebo vs. HFD + celecoxib, $3.5 \pm 0.4$ vs. $1.4 \pm 0.9$; $\mathrm{P}<0.05)$ and inflammation score (HFD + placebo vs. HFD + celecoxib, $2.8 \pm 0.4$ vs. $0.8 \pm 0.5 ; \mathrm{P}<0.05)$ (Table I).

To further demonstrate the effect of celecoxib on hepatic steatosis, a triglyceride test kit was used to examine the TG content of rat livers. The results revealed that TG content was significantly reduced in the HFD plus celecoxib group compared to the HFD plus placebo group $(\mathrm{P}<0.01)$ (Fig. 1C).

Effect of celecoxib on COX-2 expression in HFD rats. To evaluate the effects of celecoxib on COX-2 expression, quan- titative real-time RT-PCR and Western blot analysis were used. The result revealed that by 4 weeks, COX- 2 mRNA was 4.7-fold higher than in the controls and was 7.8-fold higher by week 8 (Fig. 3C). Celecoxib suppressed HFD-induced COX-2 mRNA in rats (HFD + placebo vs. HFD + celecoxib, $7.8 \pm 2.1$ fold vs. $2.2 \pm 0.5$ fold; $\mathrm{P}<0.05$ ) (Fig. $3 \mathrm{C}$ ).

COX-2 protein expression also increased following the administration of the HFD. Celecoxib treatment significantly decreased COX-2 protein expression compared to the HFD plus placebo group $(\mathrm{P}<0.05)$ (Fig. 3A and $\mathrm{B})$.

Effect of celecoxib on $N F-\kappa B$ activation in HFD-induced steatohepatitis. $\mathrm{NF}-\kappa \mathrm{B}$ is a transcription factor that regulates the expression of several proinflammatory cytokines 


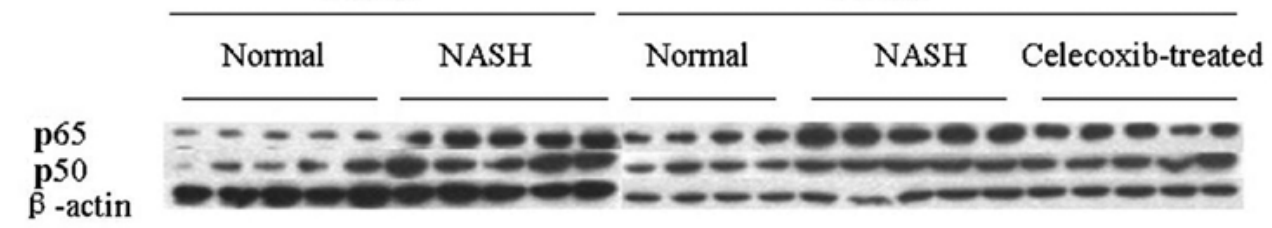

B

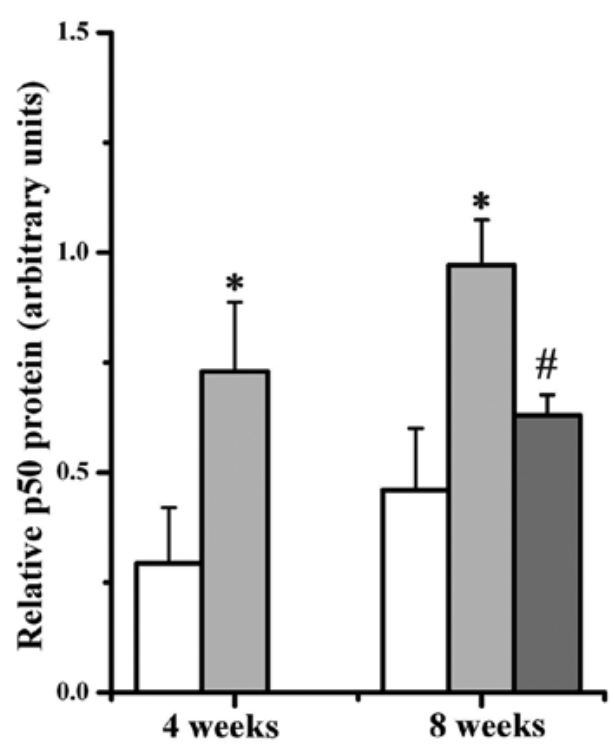

C

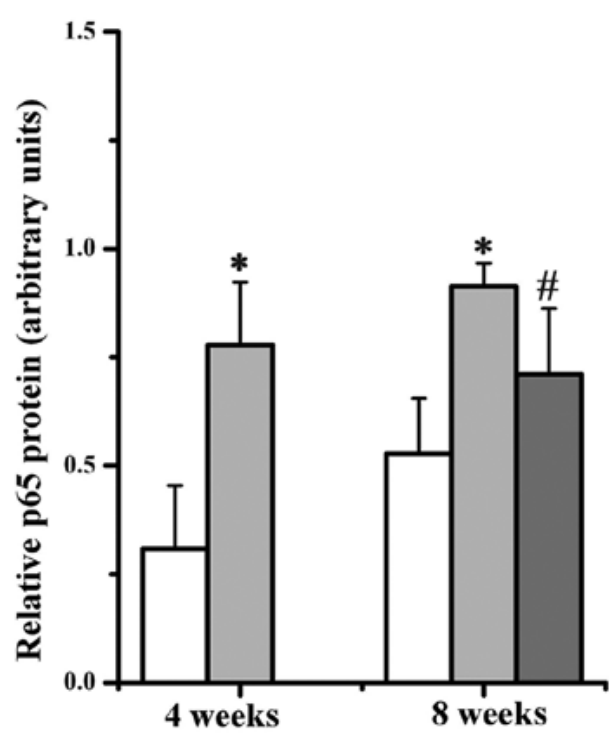

Figure 4. Expression of NF- $\kappa \mathrm{B}$ in rats. (A) Nuclear protein expression of the NF- $\kappa \mathrm{B}$ subunits, p65 and p50, was determined by Western blotting in rats fed the control diet (white bars), HFD (gray bars), or HFD with celecoxib (20 mg/kg/day) (black bars) for 4 or 8 weeks. (B and C) Bands were quantified, p65 and p50 protein levels were expressed as relative amounts normalized to $\beta$-actin. Data are expressed as the means \pm SD ( $=7-10 /$ group). $\mathrm{P}<0.05$ (HFD vs. control). ${ }^{\#} \mathrm{P}<0.05$ (HFD + celecoxib vs. HFD + placebo).

and adhesion molecules, including TNF- $\alpha$, IL-6, ICAM-1, and E-selectin. Reactive oxygen species (ROS) are common signaling molecules that drive NF- $\mathrm{NB}$ activation. Evidence has indicated that $\mathrm{NF}-\kappa \mathrm{B}$ is up-regulated both in rodent models and in patients with NASH $(12,13)$.

In our model, hepatic nuclear protein levels of NF- $\kappa \mathrm{B}$ p 65 and p50 increased significantly after 4 weeks of HFD feeding compared to the controls, and this difference was maintained until 8 weeks. Thereafter, the celecoxib coadministration down-regulated nuclear NF- $\mathrm{B}$ p65 and p50 expression significantly (Fig. 4).

\section{Discussion}

NAFLD is one of the most common chronic liver disorders, which encompasses a wide spectrum ranging from simple steatosis to cirrhosis $(14,15)$. NASH belongs to this spectrum and forms the borderline between a benign condition (steatosis) and a serious/morbid condition (cirrhosis). However, the molecular mechanisms of inflammatory recruitment that transform NAFLD to NASH remain undefined (15).

The 'two-hit' theory postulates the progression from simple steatosis to NASH, fibrosis, or cirrhosis (16). The 'first hit' consists in the accumulation of excessive hepatic fat, sensitizing the liver to the second 'hits' which lead to hepatocyte injury, inflammation and fibrosis (17). Oxidative stress-induced lipid peroxidation and cytokine-mediated injury, which have been indicated as factors of risk responsible for the 'second hit', play an important role in the liver injury occurring in NASH (18). The products of lipid peroxidation can mediate inflammatory recruitment by activating NF- $\mathrm{B}$ and COX-2 $(19,20)$. Studies have revealed that COX-2 activation in fat inflammation is crucial to the development of insulin resistance and fatty liver in high-fat-induced obese rats (21). COX-2 accumulation may be an important aspect of NASH that should receive considerable attention as a possible target for therapy. COX-2 inhibition (celecoxib) attenuates cardiovascular, liver steatosis and inflammatory aspects in monosodium glutamate-induced obese rats (22).

In this study, we report that COX-2 is markedly up-regulated during the evolution of a classical dietary model for NASH induced by feeding rats with a HFD. The temporal profile of $\mathrm{COX}-2$ and $\mathrm{NF}-\kappa \mathrm{B}$ induction coincided with increased inflammatory changes in the steatotic liver. The present data indicate that a broader role for COX-2 activation in the transition of steatosis to steatohepatitis is plausible. It was also noted that celecoxib attenuated steatosis and inflammation in HFD rats. The pharmacological effect of celecoxib was related to the suppression of $\mathrm{NF}-\kappa \mathrm{B}$.

$\mathrm{NF}-\kappa \mathrm{B}$ is a dimeric transcription factor that controls many processes including, immunity, inflammation and apoptosis. Several lines of evidence indicate that NF- $\kappa \mathrm{B}$ activation may be a critical factor leading to the development of NASH and its progression to fibrosis. The NF- $\mathrm{B}$ pathway has been found 
to be up-regulated both in rodent models and in patients with NASH (23-25). NF- $\kappa$ B has been found to activate the transcription of IL-1, IL-6, ICAM-1 and COX-2, which are involved in NASH. These cytokines could also further increase NF- $\mathrm{BB}$ activation via a feedforward mechanism (8). Evidence has revealed that the inhibition of $\mathrm{NF}-\kappa \mathrm{B}$ pathway could block liver steatosis and initiation of NASH in C57BL/6 mice (26).

The synthesis of $\mathrm{COX}-2$ is regulated by $\mathrm{NF}-\kappa \mathrm{B}$, since the promoter region of the $\mathrm{COX}-2$ gene contains two binding sites for $\mathrm{NF}-\kappa \mathrm{B}$ (27). The COX-2 inhibitor not only decreased $\mathrm{COX}-2$ but also down-regulated NF- $\mathrm{NB}$ expression through the feedforward pathway in MCD-treated mice (8). In addition to the $N F-\kappa B$ response element, the promoter region of the COX-2 gene contains binding sites for IL-1, IL- 6 and TNF- $\alpha$. Therefore, COX-2 may be another proinflammatory mediator in the transition from steatosis to steatohepatitis. In this study, we observed that celecoxib, as an anti-inflammatory drug, decreased the overexpression of COX-2 induced by HFD. Celecoxib may decrease NF- $\kappa \mathrm{B}$ p50 and p65 protein expression.

In summary, we have demonstrated that hepatic COX-2 expression is increased in HFD-induced steatohepatitis in rats. In this model, induction of COX-2 may be a consequence of $\mathrm{NF}-\kappa \mathrm{B}$ activation. The selective COX-2 inhibitor, celecoxib, may ameliorate liver injury during intake of the HFD. Another study has revealed similar results in MCD mice (8). If similar findings apply in human non-alcoholic fatty liver disease, the inhibition of COX-2 would be a logical target for new therapeutic approaches to prevent or treat non-alcoholic steatohepatitis.

However, the safety of celecoxib in humans is another problem. In our study, severe side-effects of celecoxib were not observed in Wistar rats. Although the safety of celecoxib has been approved by randomized clinical trials, certain case reports describe the hepatic toxity of celcecoxib during the treatment of non-liver diseases $(28,29)$. Although the incidence of hepatic toxity is rare, it is difficult to predict whether it will increase in the liver disease population.

\section{Acknowledgements}

This study was supported by the grant from Heilongjiang Provincial Health Department (Grant no: 2010-048) and Research Foundation of the Second Affiliated Hospital of Harbin Medical University.

\section{References}

1. Mitchell JA and Warner TD: Cyclo-oxygenase-2: pharmacology, physiology, biochemistry and relevance to NSAID therapy. Br J Pharmacol 128: 1121-1132, 1999.

2. Suleyman H, Demircan B and Karagoz Y: Anti-inflammatory and side effects of cyclooxygenase inhibitors. Pharmacol Rep 59: 247-258, 2007

3. Cheng AS, Chan HL and Leung WK, et al: Expression of HBx and COX-2 in chronic hepatitis B, cirrhosis and hepatocellular carcinoma: implication of HBx in up-regulation of COX-2. Mod Pathol 17: 1169-1179, 2004.

4. Mohammed NA, Abd El-Aleem SA, El-Hafiz HA and McMahon RF: Distribution of constitutive (COX-1) and inducible (COX-2) cyclooxygenase in postviral human liver cirrhosis: a possible role for COX-2 in the pathogenesis of liver cirrhosis. J Clin Pathol 57: 350-354, 2004.
5. Paik YH, Kim JK and Lee JI, et al: Celecoxib induces hepatic stellate cell apoptosis through inhibition of Akt activation and suppresses hepatic fibrosis in rats. Gut 58: 1517-1527, 2009.

6. Chavez E, Segovia J and Shibayama M, et al: Antifibrotic and fibrolytic properties of celecoxib in liver damage induced by carbon tetrachloride in the rat. Liver Int 30: 969-978, 2010.

7. Washino Y, Koga E and Kitamura Y, et al: Effect of celecoxib, a selective cyclooxygenase-2 inhibitor on carbon tetrachloride intoxication in rats. Biol Pharm Bull 33: 707-709, 2010.

8. Yu J, Ip E and Dela PA, et al: COX-2 induction in mice with experimental nutritional steatohepatitis: role as pro-inflammatory mediator. Hepatology 43: 826-836, 2006.

9. Larter CZ and Yeh MM: Animal models of NASH: getting both pathology and metabolic context right. J Gastroenterol Hepatol 23: 1635-1648, 2008.

10. Fan JG and Qiao L: Commonly used animal models of nonalcoholic steatohepatitis. Hepatobiliary Pancreat Dis Int 8: 233-240, 2009.

11. Zou Y, Li J, Lu C, et al: High-fat emulsion-induced rat model of non-alcoholic steatohepatitis. Life Sci 79: 1100-1107, 2006.

12. Dela PA, Leclercq I, Field J, George J, Jones B and Farrell G: NF-kappaB activation, rather than TNF, mediates hepatic inflammation in a murine dietary model of steatohepatitis. Gastroenterology 129: 1663-1674, 2005.

13. Ribeiro PS, Cortez-Pinto H, Sola S, et al: Hepatocyte apoptosis, expression of death receptors, and activation of NF-kappaB in the liver of non-alcoholic and alcoholic steatohepatitis patients. Am J Gastroenterol 99: 1708-1717, 2004.

14. Angulo P: Non-alcoholic fatty liver disease. N Engl J Med 346: 1221-1231, 2002.

15. Farrell GC and Larter CZ: Non-alcoholic fatty liver disease: from steatosis to cirrhosis. Hepatology 43: S99-S112, 2006.

16. Day CP and James OF: Steatohepatitis: a tale of two 'hits'? Gastroenterology 114: 842-845, 1998.

17. Day CP: Pathogenesis of steatohepatitis. Best Pract Res Clin Gastroenterol 16: 663-678, 2002.

18. Day CP and Saksena S: Non-alcoholic steatohepatitis: definitions and pathogenesis. J Gastroenterol Hepatol 17 (Suppl 3): 377-384, 2002.

19. Malaguarnera M, Di Rosa M, Nicoletti F and Malaguarnera L: Molecular mechanisms involved in NAFLD progression. J Mol Med 87: 679-695, 2009.

20. Leclercq IA, Farrell GC, Sempoux C, Dela PA and Horsmans Y: Curcumin inhibits NF-kappaB activation and reduces the severity of experimental steatohepatitis in mice. J Hepatol 41: 926-934, 2004

21. Hsieh PS, Jin JS, Chiang CF, Chan PC, Chen CH and Shih KC: COX-2-mediated inflammation in fat is crucial for obesitylinked insulin resistance and fatty liver. Obesity (Silver Spring) 17: 1150-1157, 2009.

22. Cunha NV, De Abreu SB, Panis C, et al: Cox-2 inhibition attenuates cardiovascular and inflammatory aspects in monosodium glutamate-induced obese rats. Life Sci 87: 375-381, 2010.

23. Yuan M, Konstantopoulos N, Lee J, et al: Reversal of obesityand diet-induced insulin resistance with salicylates or targeted disruption of Ikkbeta. Science 293: 1673-1677, 2001.

24. Crespo J, Cayon A, Fernandez-Gil P, et al: Gene expression of tumor necrosis factor alpha and TNF-receptors, p55 and p75, in non-alcoholic steatohepatitis patients. Hepatology 34: 1158-1163, 2001.

25. Videla LA, Tapia G, Rodrigo R, et al: Liver NF-kappaB and AP-1 DNA binding in obese patients. Obesity (Silver Spring) 17: 973-979, 2009.

26. Beraza N, Malato Y, Vander BS, et al: Pharmacological IKK2 inhibition blocks liver steatosis and initiation of non-alcoholic steatohepatitis. Gut 57: 655-663, 2008.

27. Pahl HL: Activators and target genes of Rel/NF-kappaB transcription factors. Oncogene 18: 6853-6866, 1999.

28. Alegria P, Lebre L and Chagas C: Celecoxib-induced cholestatic hepatotoxicity in a patient with cirrhosis. Ann Intern Med 137: 75, 2002.

29. Chamouard P, Walter P, Baumann R and Poupon R: Prolonged cholestasis associated with short-term use of celecoxib. Gastroenterol Clin Biol 29: 1286-1288, 2005. 